ARTIGO DE REVISÃO

\title{
Qualidade de vida e depressão: uma revisão da literatura
}

\author{
Quality of life and depression: a review of the literature
}

\author{
Ana Flavia Barros da Silva Lima'; Marcelo Pio de Almeida Fleck"I \\ 'Doutora. Professora, Residência Médica em Psiquiatria, Hospital Psiquiátrico São Pedro, Porto \\ Alegre, RS. \\ "Doutor. Professor adjunto, Departamento de Psiquiatria e Medicina Legal, Universidade Federal do \\ Rio Grande do Sul (UFRGS), Porto Alegre, RS. \\ Endereço para correspondência
}

\begin{abstract}
RESUMO
Os sintomas depressivos são altamente prevalentes, principalmente em serviços de cuidados primários, provocando sérios prejuízos nos sujeitos que não são submetidos a tratamento. 0 objetivo deste estudo foi o de revisar o impacto da associação entre depressão maior e qualidade de vida. Foram revisados os artigos publicados entre 1990 e 2007, utilizando as palavras-chave "qualidade de vida", "depressão", "sintomas depressivos", "serviços de cuidados primários", "bem-estar e felicidade" e "preditores de remissão". A presença de sintomas depressivos afeta todas as dimensões da qualidade de vida e, conforme a gravidade desses sintomas, o seu impacto na qualidade de vida pode ser maior do que o de outras doenças crônicas. Os resultados sugerem que a presença de sintomas depressivos exerce um importante impacto na qualidade de vida dos sujeitos, não se restringindo apenas às características clínicas do transtorno. Entretanto, ainda existe uma carência de modelos teóricos, assim como de estudos longitudinais, que possam estabelecer de forma mais clara qual é a real relação entre depressão e qualidade de vida. Provavelmente, através do entendimento de medidas subj etivas e objetivas de recuperação dos sujeitos com sintomas depressivos, poderão se buscar intervenções mais eficazes e que provoquem melhorias no funcionamento global dessa população. Sendo assim, a avaliação da qualidade de vida aparece como um desfecho relevante, pois pela sua multidimensionalidade é potencialmente capaz de detectar a magnitude e a abrangência do comprometimento que a depressão impõe.
\end{abstract}

Descritores: Qualidade de vida, depressão, serviço de cuidados primários, preditores de remissão, bem-estar, felicidade.

\footnotetext{
ABSTRACT

Depressive symptoms are highly prevalent, mainly in primary health care services, causing serious impairments in those subjects that are not treated. The objective of this study was to review the impact of the association between depressive disorder and quality of life. Our review included articles published between 1990 and 2007 and it was based on the following keywords: quality of life, depression, depressive symptoms, primary care services, well-being, happiness, and predictors of remission. The presence of depressive symptoms affects all dimensions of the quality of life, and depending on the severity of these symptoms, it might cause equivalent or more severe impairment than other chronic diseases. The findings suggest that the presence of depressive symptoms causes a serious impact on the quality of life of individuals. Such impact is not limited to the clinical characteristics of the disorder. However, there is still a lack of theoretical models and longitudinal
} 
studies demonstrating a more clear association between depression and quality of life. Most likely, through a better understanding of the subjective and objective measures of the recovery of individuals with depressive symptoms, it will be possible to find more efficient interventions that would help to improve the global functioning of this population. Therefore, the evaluation of the quality of life is a relevant outcome, since its multidimensionality is potentially capable of detecting the magnitude and scope of the impairment imposed by depression.

Keywords: Quality of life, depression, primary care services, predictors of remission, well-being, happiness.

\section{NTRODUÇÃO}

Os transtornos depressivos são muito prevalentes e estão associados com alto grau de prejuízos. Estima-se que a prevalência ao longo da vida para o transtorno depressivo maior varie entre 6 e $17 \%$, segundo dois grandes estudos epidemiológicos estadunidenses: o Epidemiological Catchment Area (ECA) e o National Comorbidity Survey, respectivamente ${ }^{1}$. Segundo os dados nacionais, há poucos estudos avaliando a prevalência desse transtorno, mas se estima que os índices variem em torno de 3 a $10 \%^{2-4}$. Cerca de $80 \%$ dos pacientes que realizam algum tipo de tratamento apresentarão um novo episódio, tendo, em média, quatro ao longo da vida, e $12 \%$ dos pacientes não apresentarão remissão total dos sintomas ${ }^{5}$. Quando comparada com outras doenças crônicas como diabetes e osteoartrites, a depressão pode provocar um comprometimento no funcionamento físico, social e ocupacional igual ou superior, independente da sua gravidade ${ }^{6-8}$. Segundo um importante estudo, o Global Burden of Disease, a depressão maior é considerada a quarta causa de incapacitação quando comparada com qualquer outra condição médica e será a segunda causa de sobrecarga em $2020^{9,10}$.

Em relação à utilização de serviços de saúde, estima-se que sujeitos com depressão excedem em até duas vezes os indivíduos sem depressão e que os custos anuais associados com o transtorno depressivo chegam a 44 bilhões de dólares nos EUA ${ }^{11-13}$. Em um estudo nacional de Fleck et al. ${ }^{14}$, os sujeitos com maior gravidade de sintomas depressivos consultavam mais nos postos de saúde, permaneciam mais tempo internados nos hospitais e faltavam mais dias ao trabalho do que os menos deprimidos.

A depressão, ao apresentar um curso crônico e recorrente, provoca prejuízos em diversas áreas da vida dos sujeitos. No entanto, a melhora dos sintomas pode não significar diretamente uma recuperação do funcionamento prévio do indivíduo. Portanto, para que se possa estabelecer o real impacto da depressão é fundamental a avaliação de desfechos multidimensionais e mais amplos que aquele centrado apenas na diminuição de sintomas.

Nas últimas décadas, tem-se constatado na Medicina um crescente interesse no estudo da percepção dos pacientes em relação a sua saúde física e mental. O parâmetro de avaliação de saúde deixou de ser centralizado nos sintomas ou na morbimortalidade, voltando-se para o bem-estar das pessoas em diversas áreas da vida. Dentro desta perspectiva, o construto "qualidade de vida" surge como um objeto de estudo em diversas áreas do conhecimento humano ${ }^{15}$. Portanto, o objetivo geral deste estudo foi revisar a associação entre qualidade de vida e depressão. Como objetivos específicos são revisados aspectos relacionados ao conceito de qualidade de vida, a aplicabilidade e limitações metodológicas do estudo deste tema, assim como o impacto da depressão na qualidade de vida.

\section{MÉTODO}

Esta revisão bibliográfica foi realizada através do sistema MEDLINE entre os anos de 1990-2007, utilizando-se as palavras-chave quality of life (qualidade de vida), depression (depressão), depressive-symptoms (sintomas depressivos), primary care (serviços de cuidados primários), well-being (bem-estar), happiness (felicidade) e remission predictors (preditores de remissão). As informações referentes a dados nacionais foram pesquisadas no sistema LILACS também no mesmo 
período de 1990-2007. Foram encontrados 3.345 artigos no MEDLINE e 18 artigos na Base de dados LILACS referentes ao assunto. Foram incluídos no estudo artigos de língua inglesa, portuguesa ou espanhola, que apresentassem sujeitos entre 18-65 anos de idade, que utilizassem alguma definição de transtornos depressivos e que se baseassem em algum construto teórico sobre qualidade de vida com a utilização de instrumentos específicos para avaliação dessa questão. Foram excluídos artigos nos quais os sujeitos apresentassem sintomas depressivos secundários a alguma condição médica, ou ensaios clínicos para avaliação da qualidade de vida em sujeitos em uso de antidepressivos. Foram obtidos 70 resumos no MEDLINE, incluindo-se 55 artigos na revisão de literatura. Dos 18 artigos encontrados na base de dados LILACS, somente dois preenchiam os critérios para inclusão no estudo.

\section{QUALI DADE DE VI DA}

O conceito de qualidade de vida

O conceito de qualidade de vida passou a ser estudado em diversas áreas do conhecimento humano no final do século XX. Em Medicina, esse estudo surgiu por uma necessidade de valorizar as percepções do paciente a respeito de vários aspectos de sua vida ao invés de meramente uma avaliação de sintomas ou de seu estado de saúde.

Apesar de a qualidade de vida ser um desfecho cada vez mais estudado na literatura, seu conceito ainda é bastante impreciso, não consensual e fundamentado em diversos modelos teóricos, o que traz problemas e limitações para o seu uso tanto na prática clínica como em pesquisa ${ }^{15}$.

\section{Modelos teóricos}

Há vários modelos teóricos que tentam fundamentar o conceito de qualidade de vida, como, por exemplo, o modelo psicológico, o modelo de preferência (utility), o modelo baseado nas necessidades do sujeito, o modelo de qualidade de vida relacionada à saúde e o modelo de Calman ${ }^{16-20}$.

O modelo psicológico baseia-se na ideia de que ter uma doença é diferente de sentir-se doente ${ }^{16}$. Por exemplo, um paciente com hipertensão arterial sistêmica possui uma doença que, na maioria das vezes, é assintomática; no entanto, a experiência de possuir uma doença pode influenciar o seu bem-estar ${ }^{16}$.

No modelo de preferência (utility), o paciente faz uma escolha entre a qualidade ou a quantidade de vida. Dois exercícios teóricos têm sido os mais usados: trade-off (troca) e standard gamble (aposta). Este modelo explora o fato de que muitos pacientes gostariam hipoteticamente de trocar a longevidade por uma qualidade de vida melhor, ou, ainda, arriscar-se diante de um procedimento pela perspectiva de poder viver melhor, sentindo-se ainda úteis ${ }^{16}$.

Um dos modelos mais utilizados é o baseado nas necessidades do sujeito. Este modelo foi implementado por Hunt \& McKenna para avaliação da qualidade de vida em pacientes com depressão. Ele postula que a vida ganha em qualidade de acordo com a habilidade e capacidade do sujeito em satisfazer as suas necessidades. A qualidade de vida é alta quando a maioria das necessidades dos seres humanos são realizadas e baixa quando poucas necessidades são satisfeitas ${ }^{17}$.

Para Calman, que estuda qualidade de vida em pacientes com câncer, a qualidade de vida só pode ser descrita e medida em termos individuais. Ele sugere que qualidade de vida é o produto da interação entre as expectativas e realizações de um indivíduo ${ }^{18}$. Assim, quanto menor for a capacidade de um indivíduo para realizar suas expectativas, mais pobre será a sua qualidade de vida. O autor ainda salienta que a ligação entre as expectativas e as realizações pode mudar ao longo do tempo, de acordo com a melhora ou a progressão da doença no indivíduo ${ }^{18}$. 
O modelo de qualidade de vida relacionada à saúde foi desenvolvido por Parsons que o definiu como "um estado de capacidade absoluta para a realização de tarefas"19. Neste modelo, a doença só se torna um problema quando ela afeta a capacidade de desempenho e a saúde é considerada como o mais valioso estado de existência ${ }^{19}$. Este modelo é baseado na capacidade funcional do sujeito, fornecendo informações sobre o nível de prejuízos ou incapacitações experienciadas para o desempenho em atividades diárias de acordo com a idade e com os principais papéis sociais no contexto de vida do sujeito ${ }^{20,21}$.

\section{Definições}

A variedade de definições de qualidade de vida contida na literatura advém, em parte, do fato de que os diferentes autores, explícita ou implicitamente, alinham-se com algum desses modelos teóricos. Além disso, existem alguns construtos vizinhos ao de qualidade de vida, sendo que as fronteiras entre eles ainda não estão claramente definidas. Condição ou estado de saúde, bem-estar e qualidade de vida são termos utilizados como sinônimos, mas que apresentam significados diferentes. O estado de saúde relaciona-se com prejuízos e incapacitações, direcionando-se a uma perspectiva mais funcional do sujeito, enquanto o bem-estar é entendido a partir do estado psicológico ou emocional, não sendo consideradas nem a dimensão cognitiva, nem a satisfação ou as condições objetivas de vida ${ }^{22}$. Apesar da diversidade de modelos conceituais para qualidade de vida e dos poucos dados empíricos que testaram a adequação desses modelos, alguns aspectos são consensuais na literatura:

1. É uma medida que varia ao longo do tempo ${ }^{16}$.

2. É uma medida subjetiva, que parte da percepção do paciente ${ }^{16}$.

3. O paciente serve como seu próprio controle, ou seja, são detectadas as mudanças que ocorrem na sua qualidade de vida de acordo com a sua trajetória de doença, ao invés de uma avaliação de ausência ou de presença de sintomas ${ }^{16}$.

4. A qualidade de vida deve ser avaliada em vários níveis: desde a avaliação do bem-estar global até domínios específicos ${ }^{16,22}$.

Medidas de qualidade de vida vêm sendo amplamente utilizadas em várias áreas médicas, em especial a Oncologia, a Reumatologia e a Cardiologia. Neste estudo é de interesse sua aplicação na área da Psiquiatria.

\section{Qualidade de vida em Psiquiatria}

A avaliação de qualidade de vida em pacientes psiquiátricos compreende diversos aspectos e possui vários desafios, como, por exemplo: qual é a importância da capacidade de juízo crítico para a avaliação da qualidade de vida nestes pacientes? Qual é o impacto na qualidade de vida dos transtornos mentais em relação ao produzido por outras condições crônicas?

Pacientes psiquiátricos que estão com as suas funções mentais prejudicadas possuem condições para avaliar sua qualidade de vida?

Embora a avaliação da qualidade de vida seja influenciada pelos acontecimentos externos, ela é uma experiência fundamentalmente subjetiva, influenciada pelo estado mental, pela personalidade e pelas expectativas de cada um ${ }^{23}$. Em relação aos pacientes que apresentam algum transtorno mental, a literatura ainda não é conclusiva quanto à validade ou não desta medida. Entretanto, parece que fatores como gravidade da doença, assim como capacidade de juízo crítico são fundamentais para direcionar se os pacientes apresentam ou não condições para a avaliação da qualidade de vida ${ }^{23}$. Por exemplo, um paciente com humor deprimido tem uma visão negativa de si, 
do mundo e do futuro, apresentando uma tendência a se sentir insatisfeito com a maior parte dos aspectos de sua vida. Entretanto, na medida em que consideramos que qualidade de vida é um construto subjetivo, o ponto de vista do paciente deve ser válido. Por outro lado, existem relativamente poucos estudos empíricos naquelas doenças mentais nas quais a capacidade de julgamento está prejudicada, como nos quadros demenciais e nas psicoses, não apresentando dados conclusivos. Alguns estudos mostram que, provavelmente, a percepção subjetiva da qualidade de vida tenha pouca validade nessa população, enquanto outros sugerem que quadros demenciais leves a moderados ou pacientes com diagnóstico de esquizofrenia, apesar de prejuízos na sua capacidade de julgamento crítico, são capazes de avaliarem a sua qualidade de vida ${ }^{23-25}$.

Os transtornos mentais provocam um impacto maior na qualidade de vida do que outras condições médicas?

Spitzer et al. compararam a qualidade de vida relacionada à saúde em pacientes com transtornos psiquiátricos ou outras condições médicas que procuravam serviços de saúde primários ${ }^{26}$. As doenças mentais, particularmente a depressão, contribuíram para maiores prejuízos na qualidade de vida em todos os domínios do SF-20 (versão abreviada do SF-36) do que outras condições médicas ${ }^{26}$. Os transtornos mentais também parecem provocar mais prejuízos em tarefas diárias do que doenças cardíacas, artrite, hipertensão e diabetes. Por outro lado, há evidências de que os diferentes diagnósticos psiquiátricos podem interferir nos diversos domínios de qualidade de vida de forma desigual. Por exemplo, os transtornos somatoformes estão mais associados a prejuízos no funcionamento de papéis, dor corporal e percepção geral da saúde; os transtornos alimentares estão mais associados a prejuízos no funcionamento social e na dor; e a depressão afeta globalmente os domínios $^{26}$. Outro aspecto relevante foi a constatação que pacientes que apresentam comorbidade psiquiátrica, ou seja, dois ou mais transtornos, apresentavam piores escores de qualidade de vida do que aqueles com diagnóstico psiquiátrico puro ${ }^{26-28}$.

Quanto aos transtornos depressivos, eles são dos transtornos mais estudados dentre os transtornos psiquiátricos em relação à qualidade de vida. Entretanto, o entendimento dessa associação ainda é muito complexo e depende de vários fatores que ainda não estão completamente bem estabelecidos pela pesquisas.

\section{Qualidade de vida e depressão}

Na literatura, encontram-se vários estudos demonstrando a existência de uma associação entre qualidade de vida e depressão. Dentre as variáveis mais estudadas encontram-se as relacionadas ao impacto da depressão na qualidade de vida, dados comparativos dos escores de qualidade de vida em sujeitos deprimidos ou com outras condições clínicas, assim como a discussão sobre se os conceitos de qualidade de vida e depressão são sobrepostos ou distintos. A seguir, estão descritos os dados da literatura referentes a essas questões.

\section{Impacto da depressão na qualidade de vida}

Os resultados sugerem que o transtorno depressivo afeta todas as dimensões da qualidade de vida, mesmo quando controlado com outras variáveis como a idade ${ }^{29,30}$. Segundo o ECA, sujeitos com depressão maior ou subssindrômica apresentam níveis maiores de tensão em atividades domésticas, assim como irritabilidade social, estresse financeiro, limitações no funcionamento ocupacional, pior status de saúde e mais dias perdidos de trabalho do que sujeitos sem sintomas ${ }^{31}$. Esses dados são replicados pelo National Comorbity Survey que demonstrou que sujeitos com transtornos de humor possuem menor escolaridade, têm um número maior de gestações na adolescência e se divorciam mais do que sujeitos sem estes transtornos ${ }^{32-34}$. 
Qualidade de vida em sujeitos com depressão e/ou outras condições clínicas

Em relação a outras condições médicas crônicas, a depressão pode acarretar maiores prejuízos na qualidade de vida e no funcionamento social, com um comprometimento que pode ser considerado 23 vezes maior do que aquele causado por outras doenças físicas ${ }^{28,35-37}$. Em relação ao status de saúde, a presença de sintomas depressivos pode exercer um impacto maior na qualidade de vida do que o sujeito se considerar saudável ou doente, ou ser maior do que presença de sintomas físicos em pessoas HIV $+{ }^{38,39}$. Num estudo populacional, Isacson et al., demonstraram que sujeitos com depressão pontuam as escalas de qualidade de vida relacionada à saúde, como a de estado de saúde (utility), com escores menores do que a população em geral ou com outras condições médicas, demonstrando o forte impacto e a sobrecarga desse transtorno na sua qualidade de vida desses sujeitos ${ }^{40}$. Outro fator relevante é que quanto maior é a intensidade de sintomas depressivos, pior é a avaliação de saúde em pacientes com alguma condição médica crônica em unidades de serviços de cuidados primários ${ }^{14}$.

\section{A associação entre depressão e qualidade de vida}

Ainda não existe um consenso na literatura quanto a se a associação entre depressão e qualidade de vida ocorre de fato ou se há uma sobreposição entre essas medidas. Em relação aos fatores comuns a ambas as medidas, há três aspectos a serem considerados. Primeiro, tanto a conceituação de depressão quanto a de qualidade de vida estão baseadas em um modelo teórico de bem-estar/satisfação, o que poderia provocar uma intersecção entre os dois construtos ${ }^{23,41}$. Entretanto, há uma carência de dados empíricos que comprovem esses modelos teóricos, provocando definições muito vagas e algumas vezes confusas $21,42,43$. Segundo, a depressão causa uma influência negativa na percepção da qualidade de vida, o que pode superestimar a relação entre elas, causando uma "falácia afetiva"44-46. Terceiro, os instrumentos de qualidade de vida, frequentemente, incluem itens que avaliam psicopatologia, ou seja, a presença de sintomas depressivos, em vez de itens específicos de qualidade de vida. Essa situação determina a ocorrência de correlações entre essas medidas que podem ser redundantes ou espúrias, uma vez que podem ocorrer mais por sobreposição de itens do que por uma associação de fato ${ }^{45}$.

Encontram-se alguns trabalhos demonstrando as semelhanças entre os referidos construtos pelo grau de correlação e simetria entre as suas variáveis. Estudos de variância mostram que as medidas de qualidade de vida contribuem com $31,5 \%$ da variância dos escores de depressão e a depressão contribui com $49 \%$ da variância nos escores de qualidade de vida 47,48 . Estes dados sugerem que a aferição da qualidade de vida apresenta um componente afetivo, conforme descrito por Heinonen et al. em estudo no qual o domínio de bem-estar emocional contribuiu com $20 \%$ da variância na avaliação dos escores de qualidade de vida global $^{49}$.

Por outro lado, existem evidências empíricas de que a qualidade de vida dos sujeitos deprimidos não está somente associada à presença de sintomas depressivos, mas também, provavelmente, a variáveis psicológicas como autoestima, coping em relação ao humor depressivo, subtipos de depressão, dimensões de personalidade e suporte social, o que sugere que depressão e qualidade de vida são conceitos correlacionados, mas distintos ${ }^{1,46,50-52}$. Rapaport et al. salientam que apesar de sujeitos com transtornos depressivos apresentarem importantes prejuízos em relação à qualidade de vida quando comparados a sujeitos sem doença, a variável específica aos sintomas contribui com uma pequena variância no modelo de regressão (em torno de 1,38\%) para o impacto na qualidade de vida, sugerindo que esses modelos devem ser semi-independentes ${ }^{50}$.

Outras evidências que sugerem que depressão e qualidade de vida representam construtos diferentes estão relacionadas com a ausência de sincronia de mudança entre os sintomas da depressão e a medida da qualidade de vida. A redução nos sintomas depressivos não se traduz necessariamente em uma melhora na qualidade de vida em todos os domínios. Em pacientes distímicos, o alívio dos sintomas pode trazer uma melhora no funcionamento social e ocupacional, mas o prazer envolvido em atividades de lazer pode permanecer prejudicados ${ }^{53,54}$. Segundo Papakostas et al., uma recente revisão, há vários estudos demonstrando que o tratamento dos sintomas depressivos na fase aguda provoca melhorias no funcionamento psicossocial e na qualidade de vida ${ }^{52}$. Estudos em fase de continuação e de manutenção demonstram mudanças menos 
acentuadas $^{52}$.

Portanto, os autores entendem que apesar de a gravidade da depressão ser um aspecto relevante de impacto na qualidade de vida, o construto de qualidade de vida não deve ser compreendido como uma sobreposição com o construto de depressão, mas sim como um construto que é determinado multifatorialmente ${ }^{51}$.

A despeito de muitas questões referentes a este tema ainda permanecerem inconclusivas, há um consenso na literatura quanto a que a associação entre depressão e qualidade de vida representa um impacto na vida dos sujeitos, sendo relevante o entendimento da aplicabilidade do conceito de qualidade de vida na prática médica assim como das limitações metodológicas envolvidas no estudo dessa medida.

\section{APLI CABI LI DADE E LI MI TAÇÕES METODOLÓGI CAS NA AVALI AÇÃO DA ASSOCI AÇÃO ENTRE QUALI DADE DE VI DA E DEPRESSÃO}

O surgimento do conceito de qualidade de vida leva a um novo paradigma na Medicina, uma vez que possibilita uma visão mais abrangente de diversos aspectos da vida dos sujeitos, não se restringindo à ausência ou presença de sintomas. Também provoca uma discussão em torno da definição de conceitos como "saúde", "doença", "bem-estar" e "satisfação". Infelizmente, a literatura ainda não é clara e conclusiva quanto à definição dessas medidas, mas nem por isso elas perdem a sua utilidade para a avaliação das pessoas ${ }^{22}$. Por exemplo, em relação à medida de qualidade de vida, ela pode servir como um parâmetro de escolha para uma melhor intervenção quando dois tratamentos são muito semelhantes no relativo à mortalidade e à morbidade ${ }^{23}$. Outra aplicabilidade relevante desta medida também pode ser aplicada para avaliar avaliação dos resultados de tratamentos de transtornos depressivos.

Apesar dos avanços obtidos nos últimos anos e da variedade de medicações disponíveis para a depressão, as respostas parciais ou inadequadas ao tratamento ainda são um evento comum ${ }^{51}$. Os estudos demonstram o perfil seguro do uso de inibidores da recaptação da serotonina, superior ao dos antidepressivos tricíclicos. Entretanto, menos de 50\% dos pacientes com depressão maior completam 6 meses de acompanhamento ${ }^{52}$. Então, por que apesar da melhora dos agentes terapêuticos ainda existe um número substancial de pacientes deprimidos que abandonam fases mais extensas ou ensaios clínicos de follow-up? Muitos aspectos estão envolvidos na avaliação desses pacientes, dentre os quais desfechos como resposta, remissão ou recuperação estão entre os mais avaliados.

A despeito da utilidade das referidas medidas, não há uma definição consensual de depressão e qualidade de vida na literatura. Os diferentes termos acabam sendo usados como se fossem sinônimos, levando a interpretações errôneas ou confusas sobre a força e a direção dos preditores relacionados ao desfecho ${ }^{51}$. Por exemplo, se for utilizado o escore final da Hamilton Depression Rating Scale (HDRS-17) como a variável em estudo, a gravidade da depressão é considerada um forte preditor de pobre resposta ao tratamento. No entanto, se a redução nos sintomas medidos pela HDRS-17 passar a ser considerada como resposta ao tratamento, em vez do escore final, é a gravidade da depressão a que passa a ser um fator preditivo positivo de moderada a boa resposta ${ }^{53}$. Portanto, a utilização de um instrumento de qualidade de vida pode ser útil nessas circunstâncias, pois acrescenta um parâmetro mais amplo não baseado em sintomas à avaliação de resposta ao tratamento ${ }^{16}$. Apesar da aplicabilidade do conceito e do fato de a associação entre qualidade de vida e depressão já estar bem demonstrada na literatura, ainda existem algumas limitações para pesquisas com este tema.

Primeiro, não existe uma definição única, clara e universal para qualidade de vida ${ }^{55}$. Segundo Gill \& Feinstein, em 1994, em um estudo para avaliar a qualidade dos artigos relacionados ao tema, somente $15 \%$ dos artigos apresentavam uma definição do conceito de qualidade de vida, havendo 159 instrumentos diferentes, dos quais 136 só haviam sido utilizados uma única vez ${ }^{56}$. Segundo, é controversa a possibilidade de se criar e operacionalizar o conceito de qualidade de vida dentro de uma perspectiva de fato universal e transcultural. A construção da maior parte dos instrumentos de qualidade de vida é realizada com influências culturais pelos pesquisadores que os criaram ${ }^{57}$. 
Terceiro, o estabelecimento de um ponto de corte e a capacidade de generalização dos resultados também estão comprometidos, uma vez que as características demográficas, doenças presentes, tipos de serviços de saúde disponíveis podem ser diferentes em áreas a serem pesquisadas em relação aos locais de criação dos instrumentos, podendo haver diferenças culturais não passíveis de comparações $^{58}$. Por último, há uma ampla discussão na literatura quanto a se a associação entre qualidade de vida e depressão de fato ocorre por uma correlação forte entre as duas medidas, ou se há uma sobreposição entre esses construtos, o que levaria a correlações espúrias ou falsas ${ }^{59,60}$. Infelizmente, os dados ainda não são conclusivos quanto a essa questão. De qualquer maneira, o conceito de qualidade de vida passa a ser um meio de ampliar a avaliação de recuperação dos sujeitos com depressão. A recuperação não pode ser entendida, somente, pelos índices de remissão ou resposta a um determinado tratamento. Mediante esses itens não é possível aferir o impacto da presença de sintomas residuais, a qualidade desses sintomas ou de outros fatores envolvidos nesse processo (como a capacidade de retornar para as atividades sociais ou profissionais) ${ }^{61,62}$. Portanto, apesar das dificuldades citadas acima, a avaliação da qualidade de vida é uma medida relevante para a avaliação de sujeitos com depressão.

\section{CONCLUSÃO}

Os resultados sugerem que a presença de sintomas depressivos exerce um importante impacto na qualidade de vida dos sujeitos, não se restringindo apenas às características clínicas do transtorno. Sendo assim, a avaliação da qualidade de vida aparece como um desfecho relevante, pois pela sua multidimensionalidade é potencialmente capaz de detectar a magnitude e a abrangência do comprometimento que a depressão impõe. Entretanto, ainda existe uma carência de modelos teóricos, assim como de estudos longitudinais, que possam estabelecer de forma mais clara qual é a real relação entre depressão e qualidade de vida. Provavelmente, pelo entendimento de medidas subjetivas e objetivas de recuperação dos sujeitos com sintomas depressivos, poderão se buscar intervenções mais eficazes e que provoquem melhorias no funcionamento global dessa população.

\section{REFERÊNCIAS}

1. Blazer DG. Mood disorders: epidemiology. In: Kaplan HI, Sadock BJ. Comprehensive of 6th ed. Philadelphia: Williams \& Wilkins; 1995. p. 1079-88.

2. Almeida-Filho N, Mari JJ, Coutinho E, França JF, Fernandes J, Andreoli SB, et al. Brazilian multicentric study of psychiatric morbidity. Methodological features and prevalence estimates. $\mathrm{Br} \mathrm{J}$ Psychiatry. 1997;171:524-9.

3. Villano LA. Problemas psicológicos e morbidade psiquiátrica em serviços de saúde não-psiquiátricos: o ambulatório de clínica geral [dissertação]. São Paulo: Escola Paulista de Medicina; 1998.

4. Fraguas RA, Ferraz TC. Depressão no Hospital Geral: estudo de 136 casos. Rev Assoc Med Bras. 1992;48(3):225-30.

5. Fleck MP, Lafer B, Sougey EB, Del Porto JA, Brasil MA, Juruena MF. Diretrizes da Associação Médica Brasileira para o tratamento da depressão. Rev Bras Psiquiatr. 2003;25(2):114-22.

6. Hays RD, Wells KB, Sherbourne CD, Rogers W, Spritzer K. Functioning and well-being outcomes of patients with depression compared with chronic general medical illnesses. Arch Gen Psychiatry. 1995;52(1): 11-9.

7. Spitzer RL, Kroenke K, Linzer M, Hahn SR, Williams JB, deGruy FV 3rd, et al. Health-related quality of life in primary care patients with mental disorders. Results from the PRIME-MD 1000 Study. JAMA. 1995;274(19): 1511-7.

8. Judd LL, Paulus MP, Wells KB, Rapaport MH. Socioeconomic burden of subsyndromal depressive symptoms and major depression in a sample of the general population. Am J Psychiatry. 
1996;153(11): 1411-7.

9. Murray CJ, Lopez AD. Global mortality, disability, and the contribution of risk factors: global burden of disease study. Lancet. 1997;349(9063):1436-42.

10. Murray CJ, Lopez AD. Alternative projections of mortality and disability by cause 1990-2020: global burden of disease study. Lancet. 1997;349(9064):1498-504.

11. Wells KB, Golding JM, Burnam MA. Psychiatric disorder in a sample of the general population with and without chronic medical conditions. Am J Psychiatry. 1988; 145(8):976-81.

12. Johnson J, Weissman MM, Klerman GL. Service utilization and social morbidity associated with depressive symptoms in the community. JAMA. 1992;267(11):1478-83.

13. Greenberg PE, Stiglin LE, Finkelstein SN, Berndt ER. The economic burden of depression in 1990. J Clin Psychiatry. 1993;54(11):405-18.

14. Fleck MP, Lima AF, Louzada S, Schestasky G, Henriques A, Borges VR, et al. [Association of depressive symptoms and social functioning in primary care service, Brazil]. Rev Saude Publica. $2002 ; 36(4): 431-8$.

15. Doward LC, McKenna SP. Evolution of quality of life assessment. In: Rajagopalan R, Sheretz EF, Anderson RT, ed. Care management of skin diseases: life quality and economic impact. New York: Marcel Dekker; 1997. p. 9-33.

16. Schipper H, Clinch J, Olweny CL. Quality of life studies: definitions and conceptual issues. In: Spilker B, ed. Quality of life and Pharmaeconomics in clinical Trials. 2nd ed. Philadelphia: LippincottRaven; 1996. p. 11-23.

17. Hunt SM, McKenna SP. The QLDS: A scale for the measurument of quality of life in depression. Health policy. 1992;22:307-19.

18. Calman KC. Quality of life in cancer patients. J Med Ethics. 1984;10:124-7.

19. Parsons T. Definition of health and illness in the light of Americam Values and social structure. In: Gartly JE, ed. Patients, physicians and ilness: a sourcebook in behavioural science and health. New York: Free Press; 1958. p. 165-87.

20. Kaplan RM, Anderson JP, Wu AW, Matthews WC, Kozin F, Oreistein D. The quality of well-being scale. Applications in AIDS, cystic fibrosis and arthritis. Medical Care. 1989;27:27-43.

21. Leplége A, Hunt S. The problem of quality of life in medicine. JAMA. 1997;278:47-50.

22. Guyatt G, Feeny D, Patrick L. Measuring health related quality of life. Ann Intern Med. 1993; 118:622-9.

23. Orley J, Saxena S, Herrman H. Quality of life and mental illness: reflections from the perspective of the WHOQOL. Br J Psychiatry. 1998;172:291-3.

24. Mozley CG, Huxley P, Sutcliffe C, Bagley H, Burns A, Challis D, et al. "Not knowing where I am doesn't mean I don't know what I like": cognitive impairment and quality of life in eldery people. Int J Geriatr Psychiatry. 1999; 14(9): 776-83.

25. Heslegrave RJ, Award AG, Vorunganti LN. The influence of neurocognitive deficits and symptoms on quality of life in schizophrenia. J Psychiatry Neurosci. 1997;22(4):235-43.

26. Spitzer RL, Kroenke K, Linzer M, Hahn SR, Williams JB, deGruy FV, et al. Health related quality of life in primary care patients with mental disorders. JAMA. 1995;274 Suppl 19:1511-7.

27. Meyer C, Rumpf HJ, Hapke U, John U. Impact of psychiatric disorders in the general population: satisfaction withlife and the influence of comorbidity and disorder duration. Soc Psychiatry Psychiatr Epidemiol. 2004;39(6):435-41. 
28. Wells KB, Sherbourne CD. Functioning and utility for current health of patients with depression or chronic medical conditions in managed, primary care practices. Arch Gen Psychiatry. 1999;56(10): 897-904.

29. Ravindran AV, Matheson K, Griffiths J, Merali Z, Anisman H. Stress, coping, uplifts, and quality of life in subtypes of depression: a conceptual frame and emerging data. J Affect Disord.

2002; 71(1-3): 121-30.

30. McCall WV, Cohen W, Reboussin B, Lawton P. Effects of mood and age on quality of life in depressed inpatients. Affect Disord. 1999;55(2-3): 107-14.

31. Johnson J, Weissman MM, Klerman GL. Service utilization and social morbidity associated with depressive symptoms in the community. JAMA. 1992;267(11):1478-83.

32. Kessler RC, Foster CL, Saunders WB, Stang PE. Social consequences of psychiatric disorders, I: Educational attainment. Am J Psychiatry. 1995; 152(7):1026-32.

33. Kessler RC, Berglund PA, Foster CL, Saunders WB, Stang PE, Walters EE. Social consequences of psychiatric disorders, II: Teenage parenthood. Am J Psychiatry. 1997; 154(10):1405-11.

34. Kessler RC, Walters EE, Forthofer MS. The social consequences of psychiatric disorders, III: probability of marital stability. Am J Psychiatry. 1998; 155(8):1092-6.

35. Spitzer RL, Kroenke K, Linzer M, Hahn SR, Williams JB, deGruy FV 3rd, et al. Health-related quality of life in primary care patients with mental disorders. Results from the PRIME-MD 1000 Study. JAMA. 1995;274(19): 1511-7.

36. Bonicatto SC, Dew MA, Zaratiegui R, Lorenzo L, Pecina P. Adult outpatients with depression: worse quality of life than in other chronic medical diseases in Argentina. Soc Sci Med. 2001;52(6):911-9.

37. Ormel J, Vonkorff M, Oldehinkel AJ, Simon G, Tiemens BG, Ustun TB. Onset of disability in depressed and non-depressed primary care patients. Psychol Med. 1999;29(4):847-53.

38. Rocha NS. Associação entre estado de saúde, espiritualidade, religiosidade e crenças pessoais e qualidade de vida [dissertação]. Porto Alegre: Universidade Federal do Rio Grande do Sul; 2002.

39. Zimpel RR. Avaliação da qualidade de vida em pacientes com HIV/AIDS [dissertação]. Porto Alegre: Universidade Federal do Rio Grande do Sul; 2003.

40. Isacson D, Bingefors K, Von Knorring L. The impact of depression is unenvely distributed in the population. European Psychiatry. 2005;20:205-12.

41. Berlim MT, Fleck MP. Quality of life and Major depression: current findings and future perspectives. In: Ritsner MS, Awad G, ed. Quality of Life Impairment in Schizophrenia, Mood and Anxiety Disorders: New Perspectives on Research and Treatment. Berlim: Springer; 2007. p. 241-52.

42. Fleck MP. Problemas conceituais em qualidade de vida. In: Fleck MP, ed. A avaliação de qualidade de vida: guia para profissionais da saúde. Porto Alegre: Artmed; 2008. p. 19-28.

43. Angermeyer MC, Kilian R. Theoretical models of quality of life mental disorders. In: Katsching $\mathrm{H}$, Freeman H, Sartorius N, ed. Quality of life in mental disorders. England: John Wiley and Sons Ltd; 1997. p. 19-30.

44. Katsching $\mathrm{H}$. How useful is the concept of quality of life in Psychiatry? In: Katsching $\mathrm{H}$, Freeman H, Sartorius N, ed. Quality of life in mental disorders. England: John Wiley and Sons; 1997. p. 3-16.

45. Katsching $\mathrm{H}$, Angermeyer MC. Quality of Life in Depression. In: Katsching $\mathrm{H}$, Freeman $\mathrm{H}$ and Sartorius N, ed. Quality of life in mental disorders. England: J ohn Wiley and Sons; 1997. p. 137-47.

46. Aigner M, Forster-Streffleur S, Prause W, Freidl M, Weiss M, Bach M. What does the 
WHOQOL-Bref measure? Measurement overlap between quality of life and depressive symptomatology in chronic somatoform pain disorder. Soc Psychiatry Psychiatr Epidemiol. 2006;41(1):81-6.

47. Godding PR, McAnulty RD, Wittrock DA, Britt DM, Khansur T. Predictors of depression among male cancer patients. J Nerv Ment Dis. 1995; 183(2): 95-8.

48. Conn VS, Taylor SG, Wiman P. Anxiety, depression, quality of life, and self-care among survivors of myocardial infarction. Issues Ment Health Nurs. 1991;12(4):321-31.

49. Heinonen $\mathrm{H}$, Aro AR, Aalto AM, Uutela A. Is the evaluation of the global quality of life determined by emotional status? Qual Life Res. 2004;13(8):1347-56.

50. Rapaport MK, Clary C, Fayard R, Endicort J. Quality of life impairment in depressive and anxiety disorders. Am J Psychiatry. 2005; 162:1171-8.

51. Kuehner C, Buerger C. Determinants of subjective quality of life in depressed patients: the role of self-esteem, response styles, and social support. J Affect Disord. 2005;86(2-3):205-13.

52. Papakostas GI, Petersen T, Mahal Y, Mischoulon D, Nierenberg AA, Fava M. Quality of life assessments in major depressive disorder: a review of the literature. Gen Hosp Psychiatry. 2004;26(1): 13-7.

53. Cassano GB, Perugi G, Maremmani I, Akiskal HS. Social adjustment in dysthymia. In: Burton SW, Akiskal HS, ed. Dysthymic disorder. London: Gaskell; 1990. p. 78-85.

54. Judd LL, Paulus MJ, Schettler PJ, Akiskal HS, Endicott J, Leon AC, et al. Does incomplete recovery from first lifetime major depressive episode herald a chronic course of illness? Am J Psychiatry. 2000;157(9):1501-4.

55. Bagby RM, Ryder AG, Cristi C. Psychosocial and clinical predictors of response to pharmacotherapy for depression. J Psychiatry Neurosci. 2002;27(4):250-7.

56. Gill T, Feintein A. A critical appraisal of the quality of quality -of-life measurements. JAMA. 1994;272 Suppl 8:619-26.

57. Sturm R, Wells KB. How can care for depression become more cost-effective? J Am Med Assoc. 1995;273:51-8.

58. Tedlow J, Fava M, Uebelacker L, Nierenberg AA, Alpert JE, Rosenbaum J. Outcome definitions and predictors in depression. Psychother Psychosom. 1998;67(4-5):266-70.

59. Rushby JF, Parker M. Culture and the measurement of health-related quality of life. Rev Européenne de psychologie Appliquée. 1995; 45 suppl 4: 257-63.

60. Whaley D, McKenna SP. Measuring quality of life in patients with depression or anxiety. Pharmaco Economics. 1995; 8 Suppl 4:305-15.

61. Fava GA, Ruini C, Belaise C. The concept of recovery in major depression. Psychol Med. 2007 Mar;37(3):307-17.

62. Ezquiaga E, Garcia-Lopez A, de Dios C, Leiva A, Bravo M, Montejo J. Clinical and psychosocial factors associated with the outcome of unipolar major depression: a one year prospective study. J Affect Disord. 2004; 79(1-3):63-70.

\section{Correspondência}

Ana Flávia Barros da Silva Lima 
Rua Mariante, 288/407

CEP 90430-180, Porto Alegre, RS

Tel.: (51) 3222.4566

E-mail: afbslima@terra.com.br

Recebido em 25/06/2007.

Aceito em 05/09/2007.

Não foram declarados conflitos de interesse associados à publicação deste artigo. 